\section{Case Reports in Neurology}

Case Rep Neurol 2021;13:65-71

DOI: 10.1159/000511957

Published online: February 1, 2021

(c) 2021 The Author(s)

Published by S. Karger AG, Basel www.karger.com/crn

This article is licensed under the Creative Commons Attribution-NonCommercial 4.0 International License (CC BY-NC) (http://www.karger.com/Services/OpenAccessLicense). Usage and distribution for commercial purposes requires written permission.

\title{
Eculizumab during Pregnancy in a Patient with Treatment-Refractory Myasthenia Gravis: A Case Report
}

\author{
Tuan Vu Brittany Harvey Niraja Suresh Jerrica Farias Clifton Gooch \\ Department of Neurology, University of South Florida, Tampa, FL, USA
}

\section{Keywords}

Generalized myasthenia gravis · Pregnancy · Eculizumab

\begin{abstract}
The recombinant humanized monoclonal antibody eculizumab has been shown to be effective and well tolerated in patients with anti-acetylcholine receptor antibody-positive, treatmentrefractory generalized myasthenia gravis (gMG). Myasthenia gravis (MG) often affects women of child-bearing potential. However, management can be challenging during pregnancy, and current treatment options are limited due to potential teratogenicity. Data are currently lacking on the use of eculizumab in pregnant women with gMG. This case report describes a successful pregnancy in a young woman with treatment-refractory gMG treated with eculizumab before, during, and after pregnancy. Eculizumab appeared to have a favorable benefit-risk profile in this setting, with no treatment-related adverse effects noted in either the patient or the neonate. The patient remains neurologically stable on eculizumab, which she has now been receiving for 5 years. This first report of the use of eculizumab during pregnancy in a patient with treatment-refractory gMG suggests a potential role for eculizumab in this setting, although further clinical experience is necessary to support its use during pregnancy in women with MG.
\end{abstract}




\section{Case Reports in Neurology}

\begin{tabular}{l|l} 
Case Rep Neurol 2021;13:65-71 \\
\hline DOI: 10.1159/000511957 & $\begin{array}{l}\text { @ 2021 The Author(s). Published by S. Karger AG, Basel } \\
\text { www.karger.com/crn }\end{array}$
\end{tabular}

Vu et al.: Eculizumab during Pregnancy in Myasthenia Gravis

\section{Introduction}

Generalized myasthenia gravis (gMG) is a chronic and debilitating autoimmune disorder characterized by fluctuating and fatigable muscle weakness. Autoantibodies directed against the nicotinic acetylcholine receptor (AChR) or other components of the neuromuscular junction have been identified as causing gMG [1]. In most patients, myasthenia gravis (MG) can be managed by treatment with the acetylcholinesterase inhibitor pyridostigmine, either alone or in combination with corticosteroids and/or other immunosuppressant drugs (e.g., methotrexate, mycophenolate mofetil, and azathioprine) [1,2]. Intravenous immunoglobulin (IVIG) or plasma exchange (PLEX) may be administered if symptoms persist $[1,2]$. However, approximately $10-15 \%$ of patients with gMG fail to respond adequately to or are intolerant of these treatment options, and their gMG is termed "refractory" [1].

MG occurs twice as frequently in women than men and typically affects women of childbearing age [3]. MG does not affect fertility, and pregnancy does not appear to have a longterm adverse impact on the course of the disease; therefore, pregnancy can be expected in women with gMG. Guidelines exist for the management of MG during pregnancy $[1,3]$. However, therapy choices are limited for use in pregnant women with refractory gMG due to the potential risk of teratogenicity with some current treatment options $[1,3]$.

The complement system plays a key role in the pathogenesis of MG [4]. The recombinant humanized monoclonal antibody eculizumab is a targeted complement inhibitor approved for the treatment of anti-AChR antibody-positive adults with gMG in the USA, refractory gMG in the EU, and gMG with symptoms that are difficult to control with high-dose IVIG therapy or PLEX in Japan [5]. Eculizumab binds to C5 complement protein and inhibits the activation of terminal complement, thereby protecting the neuromuscular junction from the effects of antibody-mediated complement activation and damage [5].

Eculizumab has been shown to be effective and well tolerated in patients with anti-AChR antibody-positive refractory gMG [5]. In the phase 3, double-blind, placebo-controlled REGAIN study, eculizumab was associated with clinically meaningful improvements in activities of daily living, muscle strength, functional ability, and health-related quality of life at 6 months [6], and these improvements were sustained through 2 years of follow-up [7]. No data are available on the use of eculizumab in women with refractory gMG during pregnancy. This case report describes a successful pregnancy in a young woman with refractory gMG who was maintained on eculizumab through her three trimesters of pregnancy.

\section{Case Report}

Data were obtained by reviewing the patient's medical records. The patient has had antiAChR antibody-positive gMG since 5-6 years of age. At a routine follow-up visit in June 2015 at 19 years of age, the patient reported new dysphagia, difficulty chewing, and episodes of choking on solid foods. She also reported continued muscle weakness, fatigue, ptosis, ophthalmoplegia with diplopia, and difficulty sleeping. She was taking pyridostigmine (120 mg every $3 \mathrm{~h}$ ). Previous treatment for MG included thymectomy, prednisone, mycophenolate mofetil, 


\section{Case Reports in Neurology}

Case Rep Neurol 2021;13:65-71

Vu et al.: Eculizumab during Pregnancy in Myasthenia Gravis

PLEX, and IVIG. Treatment with prednisone had been effective, but the patient had experienced unacceptable weight gain and tremor. She could not tolerate mycophenolate mofetil and had experienced bacteremia after PLEX. Treatment with IVIG significantly improved her strength; however, her insurance lapsed, and she was unable to fund further treatment.

On July 13, 2015 the patient was randomized to participate in the phase 3, double-blind, placebo-controlled REGAIN study (NCT01997229) [6]. At the time of entry into the study, her exam showed severe bilateral ptosis, complete external ophthalmoplegia, and mild weakness proximally in the arms (Medical Research Council [MRC] muscle strength score 4) (Table 1). Her Myasthenia Gravis Activities of Daily Living (MG-ADL) total score was 15/24, her Quantitative Myasthenia Gravis (QMG) score was 31/50, her Myasthenia Gravis Composite (MGC) score was 30/50, and her 15-item Myasthenia Gravis Quality of Life (MG-QOL15) score was $52 / 60$. For each of these instruments, higher scores are associated with worse disease severity. Based on individual score ranges, the patient's scores at baseline suggested moderately severe MG disease, with severe impact on her quality of life.

The patient completed the 6 months of double-blind treatment (placebo or eculizumab $1,200 \mathrm{mg}$ by intravenous infusion every 2 weeks) and entered the open-label extension phase. When the study closed at the end of 2018, the patient was identified as having been randomized to the active treatment arm during the initial double-blind period. She responded well to eculizumab, with rapid improvements seen on all assessment scales within 28 days of treatment initiation. She remained neurologically stable throughout the study and during the openlabel extension phase. She had no MG exacerbation or crisis during that time, compared with three relapses in the 2 years before study entry. At the end of study visit (week 108), her MGADL score was 7/24, her QMG score was 14/50, her MGC score was 11/50, and her MG-QOL15 score was $2 / 60$. Clinically significant improvements in these measures are defined as reductions of $\geq 2$ points for the MG-ADL, $\geq 2-3$ points for the QMG (based on baseline severity), $\geq 3$ points for the MGC, and $\geq 3$ points for the MG-QOL15 [8]. The patient continued to have moderate ptosis and ophthalmoplegia (with some movements, in contrast to complete ophthalmoplegia before eculizumab initiation), but limb strength normalized, and she was able to work. Thus, this patient had achieved significant improvements in disease control and quality of life with eculizumab.

At 23 years of age (about 3.5 years after the initiation of eculizumab therapy), the patient became pregnant (G1P0; approximate date of last menstrual period January 10, 2019). In February 2019, she presented with severe fatigue and exacerbation of MG symptoms; her MGADL score was 13/24. She had severe ptosis, complete ophthalmoplegia, and muscle weakness (rated MRC 4) in the hands (Table 1). She was taking pyridostigmine (120 mg every $3 \mathrm{~h}$ ), but had not been receiving eculizumab infusions regularly due to her work schedule and insurance issues. These issues were addressed, and the patient resumed regular eculizumab treatment (1,200 mg intravenous infusion over 35 min every 2 weeks). She notified the clinic of her pregnancy at the end of March and, after a discussion about treatment options, she stated that she wished to continue eculizumab treatment. During subsequent follow-up visits, the patient was found to be compliant with treatment, her MG symptoms were well controlled, and her exam observations returned to baseline. 


\section{Case Reports in Neurology}

Case Rep Neurol 2021;13:65-71

Vu et al.: Eculizumab during Pregnancy in Myasthenia Gravis

The pregnancy was uncomplicated. Regular ultrasound scans showed normal fetal development, with no apparent congenital issues. In August 2019 (32+3 weeks' gestation), the patient reported suprapubic pain lasting for 2 days and 2-3 episodes of light pink/brownish vaginal discharge. Fetal movements were reported to be normal. No vaginal bleeding or new onset/worsening of symptoms of MG were reported. Beta-hemolytic Streptococcus group B $(>100,000$ colonies) grew in her urine cultures, and she was subsequently treated with antibiotics.

At a routine visit in September 2019 (37 weeks' gestation), the patient was counseled regarding the potential for neonatal MG due to passive transmission of AChR antibodies and the need for close observation and supportive care. She remained compliant with eculizumab treatment, and her MG was well managed (MG-ADL score 6/24) (Table 1). Owing to hypertension, labor was induced at 38 weeks, resulting in vaginal delivery of a healthy female infant with a birthweight of $2.47 \mathrm{~kg}$ and an Apgar score of 9 at $5 \mathrm{~min}$.

At routine follow-up approximately 6 weeks postpartum, the patient reported worsening of MG symptoms compared with before and during pregnancy, as well as fatigue and severe headache. She was receiving ongoing treatment with pyridostigmine $60 \mathrm{mg}$ every $3 \mathrm{~h}$ and eculizumab 1,200 mg every 2 weeks. She was prescribed indomethacin $25 \mathrm{mg}$ three times daily as needed for her headaches and advised to take frequent rest. Rescue therapy was not deemed necessary at that time. The baby showed no symptoms of neonatal MG. The patient's exam showed persistent ophthalmoplegia and ptosis with normal limb strength (Table 1); her MG-ADL score was 7/24. In a telemedicine follow-up in May 2020, the patient had bilateral ptosis, almost complete ophthalmoparesis, apparently normal strength, and an MG-ADL score of $6 / 24$. She remains on eculizumab, which she has now been receiving for 5 years.

\section{Discussion}

To our knowledge, this is the first report of the use of eculizumab during pregnancy in a patient with treatment-refractory gMG. In keeping with this case report, women with MG generally have uneventful pregnancies with good outcomes [3]. Nevertheless, women with MG who are considering pregnancy should be counseled regarding the risk of complications for both themselves and their baby. As seen in our patient, disease exacerbation may occur during the first trimester and postpartum period; however, symptoms generally improve during the second and third trimesters, presumably due to the normal immunosuppressive changes that occur during late pregnancy [9].

Vaginal delivery is considered safe in pregnant women with MG and should be encouraged $[1,3]$. Because the uterus is considered a smooth muscle, its function is not thought to be severely affected by MG. The first stage of labor generally proceeds without complication. The second stage of labor requires the use of striated muscles, which may cause the patient to become tired, and use of forceps or vacuum extraction may be required to aid delivery due to prolonged labor [3].

In general, medical treatment for MG should be continued during pregnancy. Current evidence suggests that oral pyridostigmine can be safely administered throughout pregnancy in 


\section{Case Reports in Neurology}

Case Rep Neurol 2021;13:65-71

Vu et al.: Eculizumab during Pregnancy in Myasthenia Gravis

women with MG [1,3]. Prednisone is currently the immunosuppressive agent of choice during pregnancy in MG [1]. However, a slightly increased risk $(<1 \%)$ of cleft palate has been associated with first-trimester exposure to corticosteroids [3]. Other potential adverse effects associated with the use of steroids during pregnancy include higher rates of miscarriage, premature membrane rupture and preterm delivery, and lower birthweight [10]. Available evidence suggests that azathioprine and cyclosporine are relatively safe to use in pregnant women with MG who cannot tolerate steroids or whose symptoms are not satisfactorily controlled with them [1]. However, azathioprine and cyclosporine have also been associated with low birthweight and intrauterine growth retardation and are designated category D and C medications, respectively, by the Food and Drug Administration [3]. Mycophenolate mofetil and methotrexate are associated with teratogenic effects and are contraindicated during pregnancy $[3,6]$. IVIG and PLEX are useful for the management of acute exacerbations during pregnancy, but require careful monitoring of maternal and fetal wellbeing [3].

Our experience with eculizumab is in line with current evidence concerning the use of this drug during pregnancy in women with paroxysmal nocturnal hemoglobinuria (PNH), atypical hemolytic uremic syndrome (aHUS), and hemolysis, elevated liver enzymes, and low platelet count (HELLP) syndrome $[11,12]$. In a 10-year pharmacovigilance analysis in patients with PNH or aHUS, eculizumab exposure during pregnancy was reported for 434 women [12]. Pregnancy was ongoing in 136 women at the time of reporting and 38 had been lost to followup. Of the 260 pregnancies with known outcomes, 182 (70.0\%) resulted in live births. There were 41 (15.8\%) spontaneous abortions, 27 (10.4\%) induced abortions (for medical reasons or based on the patient's decision), 9 (3.5\%) stillbirths or fetal deaths, and $1(0.4 \%)$ maternal death. The overall observed rate of miscarriage was similar to the $19.7 \%$ recently reported in a cohort of 20,000 women in the United States [13]. Serious infection was reported in one neonate born to a mother treated with eculizumab; however, this event occurred 12 days after delivery and was considered by the treating physician as unlikely to be related to exposure to eculizumab [12].

As a result of passive transfer of maternal immunoglobulin $\mathrm{G}$ antibodies across the placenta during the second and third trimesters, approximately 10-20\% of infants born to mothers with MG develop transient neonatal MG [3]. This phenomenon usually resolves with support over a period of 3-4 weeks. There is no evidence to suggest any correlation between the severity of MG in the mother and the occurrence of neonatal MG [3]. Available data indicate that eculizumab treatment during pregnancy has no adverse effects on complement activity in the newborn, with no accumulation of the drug in fetal blood [14].

Breastfeeding is not contraindicated in women with MG; however, use of immunosuppressant drugs should generally be avoided. Eculizumab was not found to be present in breast milk of lactating women with PNH receiving the drug [15].

In conclusion, this case report describes a successful pregnancy in a woman with treatment-refractory gMG treated with eculizumab before, during, and after pregnancy. While eculizumab appears to have a favorable benefit-risk profile during pregnancy, further clinical experience is necessary to support the use of eculizumab during pregnancy in women with MG.

\section{Karger'=}




\section{Case Reports in Neurology}

\begin{tabular}{l|l}
\hline Case Rep Neurol 2021;13:65-71 \\
\hline DOI: 10.1159/000511957 & $\begin{array}{l}\text { (C) 2021 The Author(s). Published by S. Karger AG, Basel } \\
\text { www.karger.com/crn }\end{array}$ \\
\hline
\end{tabular}

Vu et al.: Eculizumab during Pregnancy in Myasthenia Gravis

\section{Acknowledgments}

Editorial support was provided by Jennifer Coward of Anthemis Consulting Ltd., funded by Alexion Pharmaceuticals, Inc. Alexion Pharmaceuticals, Inc. provided a medical accuracy review of the final manuscript.

\section{Statement of Ethics}

Since this is a case study involving a single patient under our care, we are exempted from Institutional Review Board approval per our local Institutional Review Board regulations. Written informed consent was obtained from the patient for publication of this case report.

\section{Conflict of Interest Statement}

T. Vu was an investigator in the REGAIN study, sponsored by Alexion Pharmaceuticals, Inc., and serves on Alexion's speakers' bureau. B. Harvey has participated in contracted services for consultation on clinical assessment operations for Alexion Pharmaceuticals, Inc. J. Farias has received personal fees for participation in a speakers' bureau for CSL Behring. N. Suresh and C. Gooch have no conflicts of interest.

\section{Funding Sources}

This research did not receive any specific grant from funding agencies in the public, commercial, or not-for-profit sectors. Editorial support was funded by Alexion Pharmaceuticals, Inc.

\section{Author Contributions}

T. Vu was the patient's primary physician. All authors contributed to the case management and to all stages of manuscript development, and approved the final version of the manuscript for submission.

\section{References}

1 Sanders DB, Wolfe GI, Benatar M, Evoli A, Gilhus NE, Illa I, et al. International consensus guidance for management of myasthenia gravis: executive summary. Neurology. 2016 Jul;87(4):419-25.

2 Farmakidis C, Pasnoor M, Dimachkie MM, Barohn RJ. Treatment of myasthenia gravis. Neurol Clin. 2018 May;36(2):311-37.

3 Waters J. Management of myasthenia gravis in pregnancy. Neurol Clin. 2019 Feb;37(1):113-20.

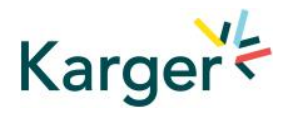




\section{Case Reports in Neurology}

\begin{tabular}{l|l}
\hline Case Rep Neurol 2021;13:65-71 \\
\hline DOI: 10.1159/000511957 & $\begin{array}{l}\text { (c) 2021 The Author(s). Published by S. Karger AG, Basel } \\
\text { www.karger.com/crn }\end{array}$ \\
\hline
\end{tabular}

Vu et al.: Eculizumab during Pregnancy in Myasthenia Gravis

4 Howard JF Jr. Myasthenia gravis: the role of complement at the neuromuscular junction. Ann N Y Acad Sci. 2018 Jan;1412(1):113-28.

5 Dhillon S. Eculizumab: A review in generalized myasthenia gravis. Drugs. 2018 Mar;78(3):367-76.

6 Howard JF Jr, Utsugisawa K, Benatar M, Murai H, Barohn RJ, Illa I, et al.; REGAIN Study Group. Safety and efficacy of eculizumab in anti-acetylcholine receptor antibody-positive refractory generalised myasthenia gravis (REGAIN): a phase 3, randomised, double-blind, placebo-controlled, multicentre study. Lancet Neurol. 2017 Dec;16(12):976-86.

7 Muppidi S, Utsugisawa K, Benatar M, Murai H, Barohn RJ, Illa I, et al.; Regain Study Group. Long-term safety and efficacy of eculizumab in generalized myasthenia gravis. Muscle Nerve. 2019 Jul;60(1):14-24.

8 Barnett C, Herbelin L, Dimachkie MM, Barohn RJ. Measuring clinical treatment response in myasthenia gravis. Neurol Clin. 2018 May;36(2):339-53.

9 Chaudhry SA, Vignarajah B, Koren G. Myasthenia gravis during pregnancy. Can Fam Physician. 2012 Dec;58(12):1346-9.

10 Gur C, Diav-Citrin O, Shechtman S, Arnon J, Ornoy A. Pregnancy outcome after first trimester exposure to corticosteroids: a prospective controlled study. Reprod Toxicol. 2004 Jan-Feb;18(1):93-101.

11 Sarno L, Tufano A, Maruotti GM, Martinelli P, Balletta MM, Russo D. Eculizumab in pregnancy: a narrative overview. J Nephrol. 2019 Feb;32(1):17-25.

12 Socié G, Caby-Tosi MP, Marantz JL, Cole A, Bedrosian CL, Gasteyger C, et al. Eculizumab in paroxysmal nocturnal haemoglobinuria and atypical haemolytic uraemic syndrome: 10 -year pharmacovigilance analysis. $\mathrm{Br}$ J Haematol. 2019 Apr;185(2):297-310.

13 Rossen LM, Ahrens KA, Branum AM. Trends in risk of pregnancy loss among US women, 1990-2011. Paediatr Perinat Epidemiol. 2018 Jan;32(1):19-29.

14 Hallstensen RF, Bergseth G, Foss S, Jæger S, Gedde-Dahl T, Holt J, et al. Eculizumab treatment during pregnancy does not affect the complement system activity of the newborn. Immunobiology. 2015 Apr;220(4):452-9.

15 Kelly RJ, Höchsmann B, Szer J, Kulasekararaj A, de Guibert S, Röth A, et al. Eculizumab in pregnant patients with paroxysmal nocturnal hemoglobinuria. N Engl J Med. 2015 Sep;373(11):1032-9. 


\section{Case Reports in Neurology}

\begin{tabular}{l|l}
\hline Case Rep Neurol 2021;13:65-71 \\
\hline DOI: 10.1159/000511957 & $\begin{array}{l}\text { (c) 2021 The Author(s). Published by S. Karger AG, Basel } \\
\text { www.karger.com/crn }\end{array}$ \\
\hline
\end{tabular}

Vu et al.: Eculizumab during Pregnancy in Myasthenia Gravis

Table 1. Findings before, during, and after pregnancy in our patient with refractory gMG

\begin{tabular}{|c|c|c|c|c|}
\hline Parameter & $\begin{array}{l}\text { June } 18,2015 \text { (before initiat- } \\
\text { ing eculizumab) }\end{array}$ & $\begin{array}{l}\text {-Pre-pregnancy (during RE- } \\
\text { GAIN¹ open-label extension) }\end{array}$ & 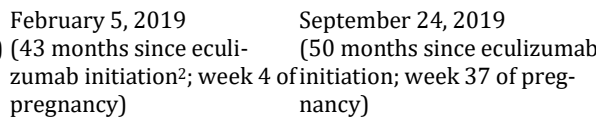 & $\begin{array}{l}\text { November } 8,2019 \\
\text { (52 months since eculi- } \\
\text { zumab initiation; } 6 \text { weeks } \\
\text { postpartum) }\end{array}$ \\
\hline Muscle strength ${ }^{3}$ & $\begin{array}{l}\text { D, B, I, Q: } 4 \text { bilaterally; } \\
\text { ADM/FDI, WE, WF, FD, FPF, } \\
\text { TE, TF: } 5 \text { bilaterally }\end{array}$ & $\begin{array}{l}\text { D, B, ADM/FDI, APB, I, Q, } \\
\text { WE, FE, FF, FD, FPF, TE, TF: } \\
5 \text { bilaterally }\end{array}$ & $\begin{array}{l}\text { ADM/FDI, APB, FE: } 4 \text { bilat- D, B, ADM/FDI, APB, I, Q, WE, } \\
\text { erally; D, B, I, Q, WE, WF, FD,FE, FF, FD, FPF, TE, TF: } 5 \text { bi- } \\
\text { FPF: } 5 \text { bilaterally } \quad \text { laterally }\end{array}$ & $\begin{array}{l}\text { D, B, ADM/FDI, APB, I, Q, } \\
\text { WE, FE, FF, FD, FPF: } 5 \text { bi- } \\
\text { laterally }\end{array}$ \\
\hline Facial weakness & lower facial weakness & none & none & none \\
\hline Speech & \multicolumn{2}{|c|}{$\begin{array}{l}\text { fluent, prosodic, nondysarth-fluent, prosodic, nondysarth-nondysarthric, } \\
\text { ric }\end{array}$} & \multicolumn{2}{|c|}{$\begin{array}{l}\text { fluent, prosodic, nondysarth- fluent, prosodic, nondys- } \\
\text { ric }\end{array}$} \\
\hline Respiration & lungs clear & $\begin{array}{l}\text { lungs clear, } \\
\text { strong cough }\end{array}$ & $\begin{array}{l}\text { RR 16; lungs clear, } \\
\text { strong cough }\end{array}$ & $\begin{array}{l}\text { lungs clear, } \\
\text { good cough effort }\end{array}$ \\
\hline Gait & normal & normal stride and base & normal stride and base & normal stride and base \\
\hline \multicolumn{5}{|c|}{$\begin{array}{l}\text { ADM/FDI, abductor digiti minimi/first dorsal interosseous; APB, abductors pollicis brevis; B, biceps; D, deltoid; FD, foot dorsiflexors; FE, finger extensors; FF, finger } \\
\text { flexors; FPF, foot plantar flexors; gMG, generalized myasthenia gravis; I, iliopsoas; Q, quadriceps; RR, respiratory rate; TE, toe extensors; TF, toe flexors; WE, wrist } \\
\text { extensors; WF, wrist flexors. }{ }^{1} \text { Phase } 3 \text {, double-blind, placebo-controlled study of eculizumab in patients with refractory gMG (NCT01997229). }{ }^{2} \text { The patient had not } \\
\text { been receiving regular eculizumab treatment for several weeks (see text). }{ }^{3} \text { Assessed using the Medical Research Council } 0-5 \text { scale for muscle strength. }\end{array}$} \\
\hline
\end{tabular}

